\title{
The 5W's for Control as Part of Industry 4.0: Why, What, Where, Who, and When-A PID and MPC Control Perspective
}

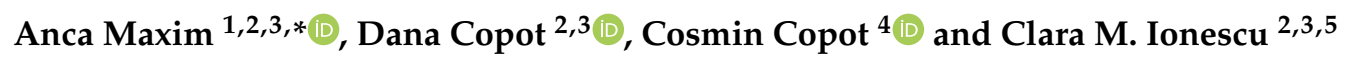 \\ 1 Department of Automatic Control and Applied Informatics, “Gheorghe Asachi” Technical University Iasi, \\ Blvd. D. Mangeron 27, 700050 Iasi, Romania \\ 2 DYSC Research Group, Ghent University, Technologiepark 914, B9052 Ghent, Belgium; \\ Dana.Copot@UGent.be (D.C.); ClaraMihaela.Ionescu@UGent.be (C.M.I.) \\ 3 Member Flanders Make Consortium, EEDT Core Group on Decision and Control, Technologiepark 914, \\ B9052 Ghent, Belgium \\ 4 OP3Mech Lab, Antwerp University, Groenenborgerlaan 171, 2020 Antwerp, Belgium; \\ cosmin.copot@uantwerpen.be \\ 5 Department of Automation, Technical University of Cluj-Napoca, Memorandumului Street 28, \\ 400114 Cluj-Napoca, Romania \\ * Correspondence: anca.maxim@ac.tuiasi.ro; Tel.: +40-751637965
}

Received: 28 December 2018; Accepted: 5 February 2019; Published: 10 February 2019

\begin{abstract}
The advent of Industry 4.0 (I4.0) has pushed technology beyond its physical limits, making the process prone to errors and poorer performance. Whether it is about smart manufacturing where mass customization is envisaged, or collaborative human-robot engineering systems, the pyramid of process operation has changed to a matrix form and control is the backbone of all process elements. The paper gives a concise guideline as to how, when, where, and what to apply when it comes to choosing the most suitable control strategy as a function of multi-parameter objective optimization. Both proportional-integral-derivative (PID) and model predictive control (MPC) control are addressed in this context.
\end{abstract}

Keywords: Industry 4.0; digital control; process automation; PID control; MPC control; monitoring; performance; robustness; process control; tuning; control design; loop interaction; scalability; networked control; wireless sensors

\section{Introduction: Why}

During the ETFA (Emerging Technologies and Factory Automation) held in Cyprus 2017, control was presented as a valuable and critical component of Industry 4.0 (I4.0) in many presentations and panel discussions with industry specialists and academics. The motivation was that merging automation with information technology requires features where control plays a key role that is vital for process performance in general. Features such as human-in-production line and plug-and-produce concepts require the presence, flexibility, and reliability of control as an intrinsic component of the new matrix approach [1]. Alas, control is present, but scarcely flexible and reliable, as concluded at the recently held industrial panel discussions at the International Federation of Automatic Control (IFAC) conference on Advances in Proportional-Integral-Derivative Control held in Belgium 2018 (www.pid18.ugent.be) and from reports on Industry in Flanders from more than 80 companies (www. flandersmake.be).

Two recently published survey papers on control in industrial applications indicated important items requiring attention from the control community [2,3]. Some of these problematic/open 
features are: networks with multiple clocks, batch processes, queuing, proportional-integral-derivative (PID)-related control structures, and the fractional order PID control. Forall these features, a common advantage of the proper structuring and tuning of controller settings is energy minimization, or equivalently, waste minimization. This is the essence of any efficient, productive management process and is one of the most important factors in the decision-making tree of allocating priorities within the matrix formulation of I4.0 and inherent control components.

The remainder of this paper is centered around the duality of using PID and MPC (model predictive control) types of control structures within the context of factory automation. Related to this is the ambiguity of how and when to re-tune controller parameters in the context of emerging changes in automation structure and challenges thereof for control technology.

\section{On the PID and MPC Control Duality: What}

Following the 2014 World Congress, IFAC launched an industry committee with the purpose of increasing industry participation and impact in its activities. A survey of the committee members' views has been consequently published in [4], where a most notable question upon the impact of specific advanced control technologies delivered a top-three result ranked in the order of their relevance: (i) PID control; (ii) MPC; and (iii) system identification. This result correlates with worldwide efforts towards I4.0 revision of the current state of practice. Of these, one may identify a core trend on data-driven analysis for structure, for performance monitoring, and for interaction assessment and mitigation.

PID control is a mature and established methodology and remains the bread and butter of a control engineer's process [5-11]. The tuning of PID controllers is efficient, yet versatile within the context of process automation. With the increasing availability of data as a result of the technology push, PID control requires revision in terms of data processing.

Data-driven structures related to control design are achieved either through virtual reference feedback tuning or fictitious reference iterative tuning [12], either revisiting basic control schemes [13,14]. In the first case, the usual algorithm consists of two steps: (i) feedback controller parameters calculated by minimizing the evaluation function relating to the disturbance response, and (ii) relating to the reference response. Efficient one-step tuning techniques are now available based on accessible data from the process, as described in [12]. An analysis on the state of use of basic process control structures reveals much room for improvement even at the PID level, particularly for feedforward control and ratio control [13]. For systems with time delays, which is a common feature in the industry, PID control parameterization in a closed loop with a predictor may be reduced to a P, PI, or PID controller with robustness specification [14]. It turns out that when a trade-off is made between IAE-based performance and M-based robustness [15], the delay error causes a disadvantage for the Smith predictor schemes, making them unusable for large values of M-based robustness. Instead, a predictive PI scheme can offer greatly improved results. In conclusion, an acclaimed method for delay-dominant processes, the Smith predictor, is shown to be outperformed by revisited PI and PID schemes with improved tuning mechanisms.

Data-driven performance monitoring to detect problems in PID loops is widely used in common factory automation architecture [6]. A plethora of software tools exist in industry for such a purpose and they are standardly used in practice. However, it was suggested that improvement may be achieved if comparable and standardized datasets could be used for testing [16]. With digitalization at hand as well as the expansion of production sites, wireless networks are a common platform for monitoring performance in factory automation. They have the advantage of flexibility and scalability when compared to wired communication. The problem of packet error rate arises from hybrid wired/wireless networks and solutions are proposed in [17] for ensuring reliability by applying machine safety standards. A special case of PID controllers are the so-called fractional order PID controllers. In [18], a study was conducted on the plant-independent evaluation of this emerging class of PID control. Some of their identified advantages were related to band-limited frequency intervals of 
specific performance specifications, as they have more tuning parameters and thus an increased degree of freedom to accomplish desired properties. Despite their very limited expansion within the industry, their potential is significant and could resolve some of the current bottlenecks $[19,20]$. However, there is much work to be done towards providing tools suitable for industrial relevance, as fractional order PID controllers are only just emerging in the control community.

Data-driven interaction assessment and mitigation for basic control loops has the advantage of not requiring the existence of an apriori model in the startup. A challenge for modeling would be the fact that interactions stem from loops with different rates. Solutions based on data availability for such situations are given in [21], thus avoiding the burden of a modeling procedure. On the fly, PID parameter tuning is achieved by a revisited tuning mechanism for dual-rate cascade control loops. Tackling a similar problem, a closed loop data-driven PID control design was proposed in [22] by minimizing a fictitious reference and ensuring robust stability of the loop.

Next to the data-driven controller design, a concept that is introduced by I4.0 as an additional term and definition is that of the Digital Twin. It is described as a tool to build highly accurate simulation models of the process. As discussed in [23], there is a clear need to disambiguate the concept. Recommendations were given to device manufacturers, system integrators, plant operators, and I4.0 architecture developers in order to clarify the terms Asset Administration Shell and Digital Twin as: (i) both terms converge against each other, with Digital Twin being an enriched synonym for the other, and (ii) the restriction of the Digital Twin on pure simulation model aspects is insufficient. These are shortlisted as:

- System integrators have to deal with the creation of interoperable digital models in the scope of their services;

- Device manufacturers need to develop and provide digital libraries of their devices including their I4.0 interfaces;

- Plant operators need to demand the use of standardized I4.0-ready components in their call for tenders.

The co-existence of data-driven control design (or re-tuning) and the Digital Twin may be exploited as a way to maintain and improve the performance monitoring of the process.

Besides PID control, there are some advanced control strategies in the industry, as summarized in [4]. There are several survey papers analyzing the use of MPC in an industrial framework [24,25].

Among research directions, the hierarchical control of plant-wide optimization is mentioned, in which at a higher layer the overall operating points of the plant (computed-based on economic optimization) are transmitted to the intermediary MPC layer. The latter computes an optimal set of reference values taking constraints and other interactions into account and relies heavily on PID controllers placed on the bottom control layer to execute short-term decisions for the actuators from the individual control loops [26-28]. The schematic representation of this strategy is given in Figure 1, where the three layers of hierarchical control are outlined with a blue dashed line, whereas the process composed of three sub-systems is marked with a green dotted line. Note that the single-headed arrows depict a unidirectional communication flow, whereas the double-headed arrows mark a bidirectional information exchange.

Another control approach is partitioning the plant into several interconnected modules, each one controlled by a local MPC which takes into account the inherent coupling and communicates with the other MPCs in order to solve its local optimization problem. This strategy is called distributed MPC (DMPC) (see review paper [29] or book [30]) and can provide an optimal or sub-optimal solution, depending on the optimization strategy employed. In Figure 2, a schematic representation of the DMPC strategy is provided. The local sub-systems are dynamically coupled, (denoted with double-headed green arrows), and are controlled by local DMPC controllers that exchange relevant information from the local optimization point of view, which are marked with double-headed blue arrows. 


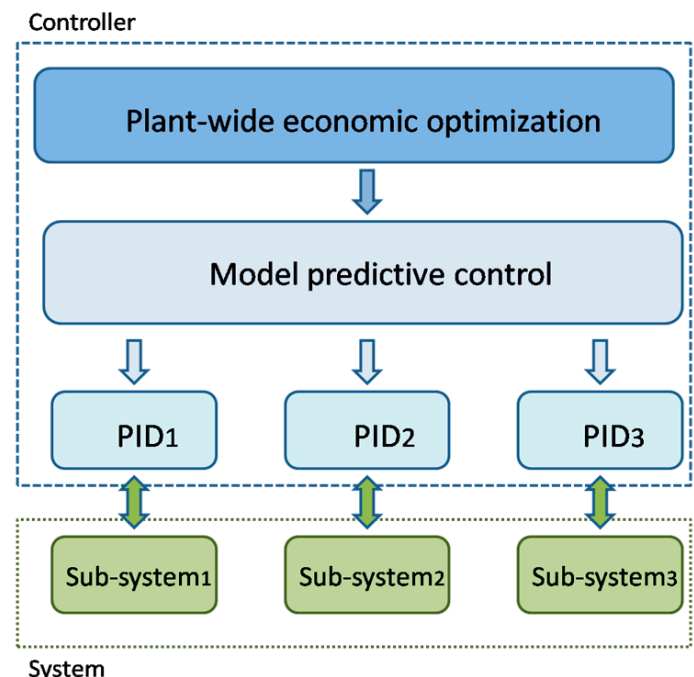

Figure 1. Schematic representation of hierarchical control.

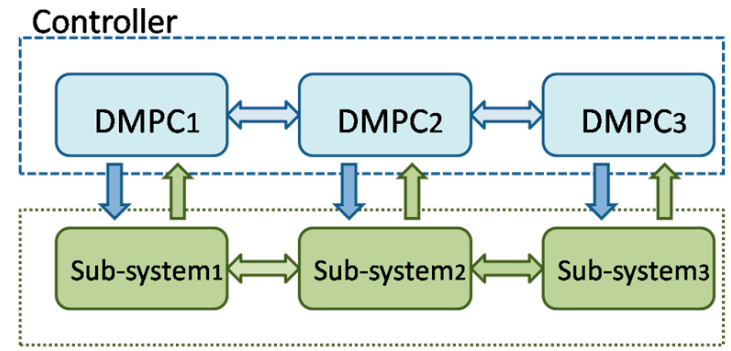

System

Figure 2. Schematic representation of the distributed model predictive control (DMPC) strategy.

Within the industrial context, an open challenge is presented by the data-based monitoring and reconfiguration of the DMPC control architecture in the presence of sensors/actuators faults [31].

\section{Where Can We Use These Tools?}

Of the application parts in the factory automation architecture, here are some listed within the context described in the previous section.

Nearly all applications of control in the process industries are regulatory control that aim to stabilize the process operation. All automation systems come with an implementation of PID controllers. Originally, the MPC applications were installed on personal computers and communicated with the base layer or PLCs via the bus system, e.g., Ethernet or OPC standards. MPC migrated to level 2 on the automation pyramid and could be easily interlinked with the base control layer of regulatory PID [32]. Manufacturing has an increasing demand for flexibility, self-optimizing plants, and human-robot cooperation. The use of collaborative robots in an automated production line is also one of the main trajectories in I4.0. A current trend in the control of such complex systems is the combination of the human-machine interaction, i.e., human-robot interaction. The MPC arises as a natural solution to predict actions from either of the players (human or machine) and optimally provide reference trajectories to each of them within the joint global optimization problem, such as path planning, force exercise, etc. A schematic representation of the human-robot interaction in the industrial environment is given in Figure 3. 


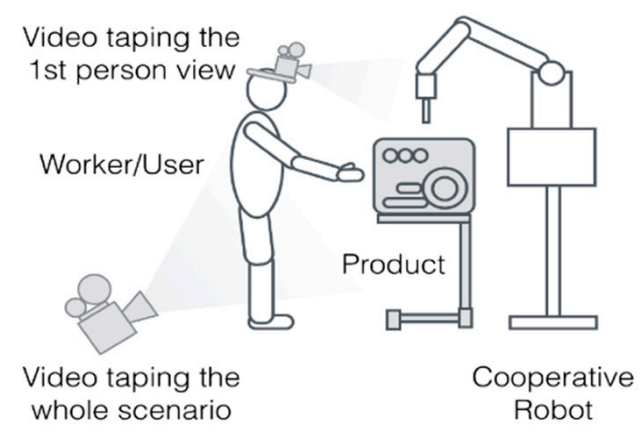

Figure 3. Schematic representation of human-robot interaction.

The changes and challenges to be tackled in I4.0 with the impact of automation technology on control technology can be summarized as follows:

- Organizational: controller parameter determined automatically; results to be represented as Key Performance Indicators (KPIs) for non-expert evaluation.

- Technological: truly distributed control in the form of simple, cheap control algorithms, based on multiple measurements that can communicate with each other.

- Technological: wireless communication that requires no equidistant sampling and no measurement available for extended periods of time.

- Technological: cloud storage with increased computational power available for historical data analysis as well as available and exchangeable data from other sources.

- Business drivers: increased need for auto-tuning for changing operating conditions.

The transition towards a cyber-physical production system is thus imminent and requires machine-to-machine connectivity and reliability [33]. Ideally, this will imply a high level of security without requiring intense modifications of the production network or production device [34,35]. Inclusive interfaces for human-machine context are also necessary tools [36]. Ideally, this is an adaptive framework as individuals with various degrees of expertise and practice may be shuffled into various segments of the production. A flexible solution to overcome the high demand in scalability and product specificity is to gradually converge to a plug and produce system of production with generic device or machine configuration and adapters [37,38]. The Digital Twin could then be easily adapted and used for the custom simulation of products $[39,40]$.

\section{The Leaders and Followers: Who}

In the industry, adaptive control is often obtained by manual ad hoc design for which no systematic tools exist to support the user. This approach has a few downsides. Firstly, it requires a good control engineer and considerable effort to realize a well-performing manually-defined adaptive controller. This follows naturally from the fact that the engineer has to use their insight to formulate the appropriate adaptation schemes. Secondly, this approach does not scale very well since the amount of effort needed for manual tuning increases significantly. Thirdly, the approach also does not lend itself to new and complex problems as insight will need to be derived first before any sort of adaptive scheme can be manually built.

From this overview, we can conclude that while we use existing robust and optimal control methods to optimize control actions, given the current context we want to make improvements with respect to the state-of-the-art and state-of-the-practice in several other ways:

- To simplify implementation by performing computationally intensive optimization offline.

- To develop tools that are generic and can be used for many different types of controllers.

- To develop controllers that adapt to variations in context; however, we cannot assume in advance that we know what context variables are relevant. 
In general, the growth of the industrial control and factory automation market is due to the increasing demand for the automation and adoption of I4.0 in manufacturing industries. However, compared to the hard automation that has driven industrial innovation in the last few decades, new automation needs to be flexible and adapt to variations in environmental conditions and to variations in products being manufactured. Therefore, the growing demand in automation goes hand-in-hand with a demand for flexible controllers.

The main industrial sectors for context-aware controllers are: machine manufacturing, machine components manufacturing, and software engineering tools developing companies. The main focus is to use and implement context-aware controllers in their products or services. The common factor within the industry is that companies are confronted with changing conditions in either production or machine operation.

Following the I4.0 paradigm shift, worldwide industry leaders present tools for softening the transition from the current to the future state of industry background. ABB proposes the use of AutomationML tools for interoperability among various other existing tools [41]. They also propose an algorithm for the automatic generation of plant topology from information received/monitored in an operations data flow [42]. Within the standard networking environment, Emerson proposes a Linux-based standard to regulate network flow within the process automation [43]. On one side it is clear that the industry pushes forward as the technology push is processed. Standardization would on one hand ease the challenges for control, while on the other hand it would also provide a global framework of communication and understanding, making evaluation and monitoring an easier task, therefore increasing transparency among players.

Important, if not vital, is the availability of student software tools for the understanding of control, i.e., PID tuning [5,8,44] and MPC tuning [45-47]. This is an important aspect for preparing future engineers for the updated context of factory automation as well as the readily at-hand tools integrated into the process control loops (e.g., auto-tuning).

\section{A Matter of Tuning: When}

According to [48], a well-tuned PID controller loses $60 \%$ of its performance after the first six months of operation. Performance monitoring is thus essential in the decision-making process of auto-tuning the base control layer in order to ensure optimal performance in the upper layers (MPC) [49]. This is schematically represented in Figure 4. Despite the broad applicability of PID control, there is the question as to when PID control is a good choice for the respective loop dynamics. A comprehensive discussion against generic linear time invariant (LTI) controllers is given in [50]. An alternative to MPC is found in the constrained predictive PID controllers that are proposed in [51], presented as a solution to overcome wireless communication-related control challenges.

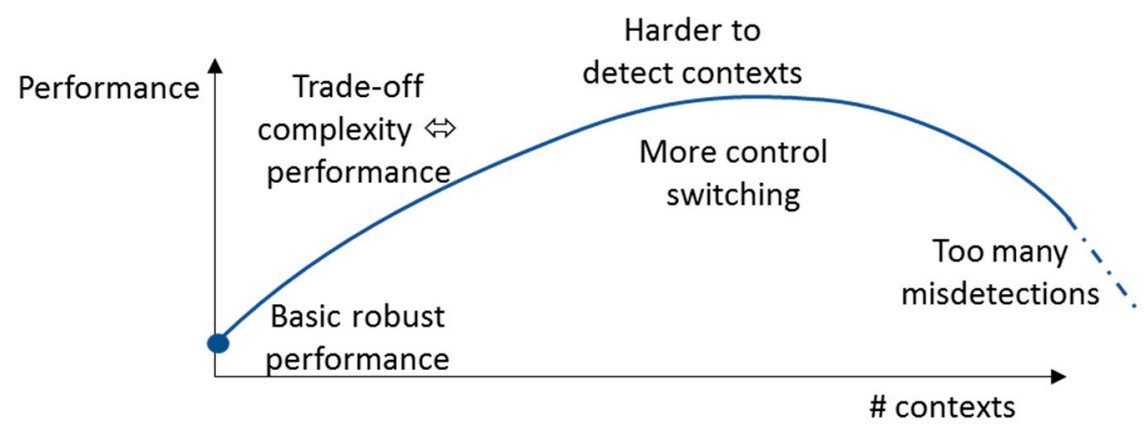

Figure 4. The performance of the context-aware controller will be optimal with the right balance between enough complexity and not too many misdetections.

Short and robust experiments are clearly preferred to long, costly tests for identifying controller parameters that are given a set of specifications for closed loop performance [52]. Recent advances in 
auto-tuning methods include both integer-order [53-56] and fractional order tuning algorithms [57]. Furthermore, an interesting decision-making algorithm for tuning controller parameter is based on event-trigger auto-tuning [54-56].

\section{Conclusions}

The aim of this paper is to give an overview of the current challenges in the industry from the control perspective. In this article, guidelines on how, when, where, and what to apply when the decision on the most suitable control strategy has to be made, while also considering the multi-parameter objective optimization. For this, we have considered both classic (PID) and advanced control (MPC). I4.0 technologies seem to be rapidly evolving, and there is room to iterate. Learning from previous experiences can inform the next set of initiatives and help home in on the next list of priorities.

Changes are happening quickly, but with change often comes opportunity-the opportunity to exploit I4.0's ability to play an integral role in strategic decision-making, broader ecosystem integration, and the customization of products and services to the specific needs of customers and clients.

Author Contributions: A.M., D.C., C.C. and C.M.I. contributed equally to the content of the review paper, writing, and editing.

Funding: This research received no external funding.

Conflicts of Interest: The authors declare no conflict of interest.

\section{References}

1. Perez Olaya, S.S.; Wollschlaeger, M. Control as an Industrie 4.0 Component. In Proceedings of the 22nd IEEE International Conference on Emerging Technologies and Factory Automation (ETFA), Limmasol, Cyprus, 12-15 September 2017. [CrossRef]

2. Leva, A. PID-based controls in computing systems: a brief survey and some research directions. In Proceedings of the 3rd IFAC Conference on Advances in Proportional-Integral-Derivative Control, Ghent, Belgium, 9-11 May 2018; pp. 805-810.

3. Tepljakov, A.; Alagoz, B.B.; Yeroglu, C.; Gonzalez, E.; HosseinNia, S.H.; Petlenkov, E. FOPID controllers and their industrial applications: a survey of recent results. In Proceedings of the 3rd IFAC Conference on Advances in Proportional-Integral-Derivative Control, Ghent, Belgium, 9-11 May 2018; pp. 25-30.

4. Samad, T. A survey on industry impact and challenges thereof. IEEE Control Syst. Mag. 2017, 37, 17-18. [CrossRef]

5. Åström, K.; Hägglund, T. Advanced PID Control; ISA: Fort Belvoir, VI, USA, 2006; ISBN 9781556179426.

6. Skilton, M.; Hovsepian, F. The 4th Industrial Revolution; Palgrave Macmillan: Basingstoke, UK; Springer Nature: Basingstoke, UK, 2018; ISBN 9783319624785.

7. Alfaro, V.M.; Vilanova, R. Model-Reference Robust Tuning of PID Controllers; Advances in Industrial Control; Springer International Publishing: Basel, Switzerland, 2016; ISBN 9873319282114.

8. Johnson, M.; Moradi, M. PID Control; Springer: London, UK, 2005; ISBN 9781852337025.

9. Visioli, A. Practical PID Control; Advances in Industrial Control; Springer: London, UK, 2006; ISBN 9781846285851.

10. Boiko, I. Non-Parametric Tuning of PID Controllers: A Modified Relay-Feedback Test Approach; Advances in Industrial Control; Springer: London, UK, 2013; ISBN 9781447144649.

11. Wang, Q.-G.; Ye, Z.; Cai, W.-J.; Hang, C.-C. PID Control for Multivariable Processes; Lecture Notes in Control and Information Sciences 373; Springer: Berlin/Heidelberg, Germany, 2008; ISBN 9783540784814.

12. Kinoshita, T.; Yamamoto, T. Design of a data-oriented PID controller for a two degree of freedom control system. In Proceedings of the 3rd IFAC Conference on Advances in Proportional-Integral-Derivative Control, Ghent, Belgium, 9-11 May 2018; pp. 412-415.

13. Hägglund, T.; Guzman, J.L. Development of basic process control structures. In Proceedings of the 3rd IFAC Conference on Advances in Proportional-Integral-Derivative Control, Ghent, Belgium, 9-11 May 2018; pp. 775-780. 
14. Grimholt, C.; Skogestad, S. Should we forget the Smith Predictor? In Proceedings of the 3rd IFAC Conference on Advances in Proportional-Integral-Derivative Control, Ghent, Belgium, 9-11 May 2018; pp. 769-774.

15. Papadopoulos, K. PID Controller Tuning Using the Magnitude Optimum Criterion; Springer International Publishing: Basel, Switzerland, 2015; ISBN 9783319072623.

16. Bauer, M.; Auret, L.; le Roux, D.; Aharonson, V. An industrial PID data repository for control loop performance monitoring (CPM). In Proceedings of the 3rd IFAC Conference on Advances in Proportional-Integral-Derivative Control, Ghent, Belgium, 9-11 May 2018; pp. 823-828.

17. Dietrich, S.; May, G.; Wetter, O.; Heeren, H.; Fohler, G. Performance indicators and use case analysis for wireless networks in factory automation. In Proceedings of the 22nd IEEE International Conference on Emerging Technologies and Factory Automation (ETFA), Limmasol, Cyprus, 12-15 September 2017. [CrossRef]

18. Alagoz, B.; Tepljakov, A.; Yeroglu, C.; Gonzalez, E.; HosseinNia, H.; Petlenkov, E. A numerical study for plant-independent evaluation of fractional-order PID controller performance. In Proceedings of the 3rd IFAC Conference on Advances in Proportional-Integral-Derivative Control, Ghent, Belgium, 9-11 May 2018; pp. 539-544.

19. Monje, C.A.; Chen, Y.Q.; Vinagre, B.M.; Xue, D.; Feliu, V. Fractional-Order Systems and Controls; Advances in Industrial Control Series; Springer: London, UK, 2010; ISBN 9781849963343.

20. Padula, F.; Visioli, A. Advances in Robust Fractional Control; Springer International Publishing: Basel, Switzerland, 2015; ISBN 9783319109299.

21. Ito, S.; Sato, T.; Araki, N.; Konishi, Y. Two-loop design for dual-rate cascade system. In Proceedings of the 3rd IFAC Conference on Advances in Proportional-Integral-Derivative Control, Ghent, Belgium, 9-11 May 2018; pp. 581-585.

22. Kurokawa, R.; Sato, T.; Vilanova, R.; Konishi, Y. Closed-loop data-driven trade-off PID control design. In Proceedings of the 3rd IFAC Conference on Advances in Proportional-Integral-Derivative Control, Ghent, Belgium, 9-11 May 2018; pp. 244-249.

23. Wagner, C.; Grothoff, J.; Epple, U.; Drath, R.; Malakuti, S.; Grüner, S.; Hoffmeister, M.; Zimmerman, P. Performance indicators and use case analysis for wireless networks in factory automation. In Proceedings of the 22nd IEEE International Conference on Emerging Technologies and Factory Automation (ETFA), Limmasol, Cyprus, 12-15 September 2017. [CrossRef]

24. Qin, S.J.; Badgwell, T.A. A survey of industrial model predictive control technology. Control Eng. Pract. 2003, 11, 733-764. [CrossRef]

25. Scattolini, R. Architectures for distributed and hierarchical Model Predictive Control—A review. J. Process Control 2009, 19, 723-731. [CrossRef]

26. Brdys, M.A.; Grochowski, M.; Gminski, T.; Konarczak, K.; Drewa, K. Hierarchical predictive control of integrated wastewater treatment systems. Control Eng. Pract. 2008, 16, 751-767. [CrossRef]

27. Tatjewski, P. Advanced control and on-line process optimization in multilayer structures. Annu. Rev. Control 2008, 32, 71-85. [CrossRef]

28. Rawlings, J.B.; Stewart, B.T. Coordinating multiple optimization-based controllers: New opportunities and challenges. J. Process Control 2008, 18, 839-847. [CrossRef]

29. Christofides, P.D.; Scattolini, R.; Muñoz de la Peña, D.; Liu, J. Distributed model predictive control: A tutorial review and future directions. Comput. Chem. Eng. 2013, 51, 21-41.

30. Maestre, J.M.; Negenborn, R.R. Distributed Model Predictive Control Made Easy; Intelligent Systems, Control and Automation: Science and Engineering; Springer: Dordrecht, The Netherlands; Heidelberg, Germany; New York, NY, USA; London, UK, 2014; Volume 69, ISBN 9789400770058.

31. Chilin, D.; Liu, J.; Davis, J.F.; Christofides, P.D. Data-based monitoring and reconfiguration of a distributed model predictive control system. Int. J. Robust Nonlinear 2012, 22, 68-88. [CrossRef]

32. Bauer, M.; Schlake, J.C. Changes to the automation architecture. In Proceedings of the 22nd IEEE International Conference on Emerging Technologies and Factory Automation (ETFA), Limmasol, Cyprus, 12-15 September 2017. [CrossRef]

33. Schmitter, C.; Ma, Z.; Ruprechter, T.; Aldrian, A. Practical safe, secure and reliable machine-to-machine connectivity for cyber-physical-production systems. In Proceedings of the 22nd IEEE International Conference on Emerging Technologies and Factory Automation (ETFA), Limmasol, Cyprus, 12-15 September 2017. [CrossRef] 
34. Ustundag, A.; Cevikcan, E. Industry 4.0: Managing the Digital Transformation; Springer Series in Advanced Manufracturing; Springer International Publishing: Basel, Switzerland, 2018; ISBN 9783319578699.

35. Thames, L.; Schaefer, D. Cybersecurity for Industry 4.0, Analysis for Design and Manufacturing; Springer Series in Advanced Manufacturing; Springer International Publishing AG: Basel, Switzerland, 2017; ISBN 9783319506593.

36. Villani, V.; Sabattini, L.; Czerniak, J.N.; Mertens, A.; Vogel-Heuser, B.; Fantuzzi, C. Towards modern inclusive factories: a methodology for the development of smart adaptive human-machine interfaces. In Proceedings of the 22nd IEEE International Conference on Emerging Technologies and Factory Automation (ETFA), Limmasol, Cyprus, 12-15 September 2017. [CrossRef]

37. Dorofeev, K.; Cheng, C.H.; Guedes, M.; Ferreira, P.; Profanter, S.; Zoitl, A. Device adapter concept towards enabling plug \& produce production environments. In Proceedings of the 22nd IEEE International Conference on Emerging Technologies and Factory Automation (ETFA), Limmasol, Cyprus, 12-15 September 2017. [CrossRef]

38. Eisenmenger, W.; Meßmer, J.; Wenger, M.; Zoitl, A. Increasing control application reusability through generic device configuration model. In Proceedings of the 22nd IEEE International Conference on Emerging Technologies and Factory Automation (ETFA), Limmasol, Cyprus, 12-15 September 2017. [CrossRef]

39. Ericsson, N.; Lennvall, T.; Åkerberg, J.; Björkman, M. Custom simulation of industrial wireless sensor and actuator network for improved efficiency during research and development. In Proceedings of the 22nd IEEE International Conference on Emerging Technologies and Factory Automation (ETFA), Limmasol, Cyprus, 12-15 September 2017. [CrossRef]

40. Brusa, E.; Cala, A.; Ferretto, D. Systems Engineering and Its Application to Industrial Product Development; Studies in Systems, Decision and Control 134; Springer International Publishing AG: Basel, Switzerland, 2018; ISBN 9783319718361.

41. Bihani, P.; Drath, R. Concept for AutomationML-based interoperability between multiple independent engineering tools without semantic harmonization. In Proceedings of the 22nd IEEE International Conference on Emerging Technologies and Factory Automation (ETFA), Limmasol, Cyprus, 12-15 September 2017. [CrossRef]

42. Gutermuth, G.; Hoernicke, M. Automatic generation of plant topologies by analyzing operations data. In Proceedings of the 22nd IEEE International Conference on Emerging Technologies and Factory Automation (ETFA), Limmasol, Cyprus, 12-15 September 2017. [CrossRef]

43. Amaro, A.; Nixon, M. Towards IEC62439-3: Implementing Linux based (ZHAW-InES) PRP stack with non-PRP passthrough on Ethernet networks in QNX. In Proceedings of the 22nd IEEE International Conference on Emerging Technologies and Factory Automation (ETFA), Limmasol, Cyprus, 12-15 September 2017. [CrossRef]

44. Rossiter, J.A. Evaluation of student software tools for supporting an understanding of PID tuning. In Proceedings of the 3rd IFAC Conference on Advances in Proportional-Integral-Derivative Control, Ghent, Belgium, 9-11 May 2018; pp. 322-327.

45. Rossiter, J.A. A First Course in Predictive Control, 2nd ed.; CRC Press: London, UK, 2018; ISBN 9781138099340.

46. Wang, L. Model Predictive Control System Design and Implementation Using MATLAB ${ }^{\circledR}$; Advances in Industrial Control Series; Springer: London, UK, 2009; ISBN 9781848823303.

47. Kouvaritakis, B.; Cannon, M. Model Predictive Control, Advanced Textbooks in Control and Signal Processing; Springer International Publishing: Basel, Switzerland, 2016; ISBN 9783319248516.

48. Bauer, M.; Horch, A.; Xie, L.; Jelali, M.; Thornhill, N. The current state of control loop performance monitoring-A survey of application in industry. J. Process Control 2016, 38, 1-10. [CrossRef]

49. Li, Y.; Lin, Z. Stability and Performance of Control Systems with Actuator Saturatio; Birkhäuser, Springer International Publishing AG: Basel, Switzerland, 2018; ISBN 9783319642444.

50. Soltesz, K.; Cervin, A. When is PID a good choice? In Proceedings of the 3rd IFAC Conference on Advances in Proportional-Integral-Derivative Control, Ghent, Belgium, 9-11 May 2018; pp. 250-255.

51. Vasquez, M.; Katebi, R. New constrained predictive PID controller for packet dropouts in wireless networked control systems. In Proceedings of the 3rd IFAC Conference on Advances in Proportional-Integral-Derivative Control, Ghent, Belgium, 9-11 May 2018; pp. 811-816.

52. Liu, T.; Gao, F. Industrial Process Identification and Control Design; Step-Test and Relay-Experiment Based Methods, Advances in Industrial Control; Springer: London, UK, 2012; ISBN 9780857299765. 
53. Berner, J.; Soltesz, K. Short and robust experiments in relay autotuners. In Proceedings of the 22nd IEEE International Conference on Emerging Technologies and Factory Automation (ETFA), Limmasol, Cyprus, 12-15 September 2017. [CrossRef]

54. Sanchez, J.; Guinaldo, M.; Visioli, A.; Dormido, S. Identification of process transfer function parameters in event-based PI control loops. ISAT 2018, 75, 157-171. [CrossRef] [PubMed]

55. Sanchez, J.; Guinaldo, M.; Visioli, A.; Dormido, S. Enhanced event-based identification procedure for process control. Eng. Chem. Res. 2018, 57, 7118-7231.

56. Merigo, L.; Beschi, M.; Padula, F.; Visioli, A. A noise-filtering event generator for PIDPlus controllers. J. Franklin Inst. 2018, 355, 774-802. [CrossRef]

57. Monje, C.A.; Vinagre, B.M.; Feliu, V.; Chen, Y. Tuning and auto-tuning of fractional order controllers for industry applications. Control Eng. Pract. 2008, 16, 798-812.

2019 by the authors. Licensee MDPI, Basel, Switzerland. This article is an open access article distributed under the terms and conditions of the Creative Commons Attribution (CC BY) license (http:/ / creativecommons.org/licenses/by/4.0/). 